\title{
Topographic abnormality of slow cortical potentials in schizophrenia
}

L.F.H. Basile, J. Yacubian, B.L.C. Ferreira, A.C. Valim and W.F. Gattaz
Laboratório de Neurosciências (LIM-27), Departamento de Psiquiatria, Faculdade de Medicina, Universidade de São Paulo, São Paulo, SP, Brasil

\author{
Correspondence \\ L.F.H. Basile \\ Laboratório de Neurosciências \\ Departamento de Psiquiatria \\ FM, USP \\ Av. Dr. Ovidio Pires de Campos, s/n \\ 05403-010 São Paulo, SP \\ Brasil \\ Fax: +51-11-3673-2201 \\ E-mail: Ibasile@usp.br
}

Research supported by FAPESP

(Nos. 98/07640-3 and 97/11083-0).

Received April 9, 2003

Accepted September 16, 2003

\begin{abstract}
A recent study from our laboratory has provided evidence for the generation of slow potentials occurring in anticipation to task-performance feedback stimuli, in multiple association cortical areas, consistently including two prefrontal areas. In the present study, we intended to determine whether these slow potentials would indicate some abnormality (topographic) in schizophrenic patients, and thus serve as an indication of abnormal association cortex activity. We recorded slow potentials while subjects performed a paired-associates memory task. A 123-channel EEG montage and common average reference were used for 20 unmedicated schizophrenic (mean duration of illness: $11.3 \pm 9.2$ years; mean number of previous hospitalizations: 1.2 \pm 1.9 ) and 22 healthy control subjects during a visual paired-associates matching task. For the topographic analysis, we used a simple index of individual topographic deviation from normality, corrected for absolute potential intensities. Slow potentials were observed in all subjects. Control subjects showed a simple spatial pattern of voltage extrema (left central positive and right prefrontal negative), whereas schizophrenic patients presented a more complex, fragmented pattern. Topographic deviation was significantly different between groups $(\mathrm{P}<0.001)$. The increased topographic complexity in schizophrenics could be visualized in grand averages computed across subjects. Increased topographic complexity could also be seen when grand averages were computed for subgroups of patients assembled either according to task-performance (high versus low) or by their scores on psychopathological scales. There was no significant correlation between topographic deviation and psychopathology scores. We conclude that the slow potential topographic abnormalities of schizophrenia indicate an abnormality in the configuration of large-scale electrical activity in association cortices.
\end{abstract}

Key words

- High-resolution EEG

- Slow potentials

- Contingent negative

variations

- Schizophrenia

- Source localization

- Functional brain mapping

\section{Introduction}

Since quantitative and averaging electroencephalographic methods were introduced, various electrophysiological alterations have been reported in schizophrenia. Alterations were found in the power of particular spectral bands of the ongoing electroencephalogram, or in latencies, intensities and topography of event-related potentials. Among the former are studies indicating increased power on delta and theta bands in schizophrenia, at 
frontal electrodes, originally considered to represent the electrical version of 'hypofrontality' $(1,2)$, although recent source localization studies showed these alterations in frontotemporal and posterior cortical areas (3). With respect to the second type of electroencephalographic studies on strictly sensory or exogenous evoked potentials, the literature is still controversial, and even findings that were considered among the most consistent in the literature (nonsuppression of the P50 auditory evoked potential) are currently being reinterpreted after the development of new methods of recording and analysis (4).

It is expected that pathophysiological studies in schizophrenia should benefit more from indexes of association rather than sensory-motor cortical activity. The analysis of endogenous potentials of the P300 class or of slow potentials (of the contingent negative variation $(\mathrm{CNV})$ type), which are phenomena more directly related to voluntary behavior, offers a possibility for study. The P300s are attention-related potentials (5) believed to originate in sensory plus polymodal cortices (6). Increased peak latencies and reduced amplitudes have been observed in schizophrenia for visual, auditory and somatosensory P300 potentials (7-11).

The slow potentials of the CNV class are particularly interesting, since they appear to reflect electrical activity from the prefrontal cortex. The CNV obtained during traditional tasks, which require a simple timing of motor responses, are negative shifts of the electroencephalogram that peak at fronto-central sites (12). However, it seems that the mere anticipation of any type of task-relevant stimulus is sufficient to provoke slow potentials. Since their discovery (13), CNVs have been assumed to be generated by the (pre)frontal cortex. Evidence from different types of studies has confirmed the prefrontal areas at least as the main centers of slow potential generation, i.e., studies with modeling of intracranial generators of CNVs or their magnetoencephalographic equivalents in the form of few equivalent current dipoles (14-18), lesion studies $(19,20)$, and invasive intracranial recordings $(21,22)$.

In a recent study, using a 123-channel electroencephalographic montage and common average reference, we analyzed the intracranial generators of the slow potentials in healthy subjects, including the control individuals that participated in the present study, by current density reconstruction. We found evidence for multifocal generation of these slow potentials, including prefrontal areas 9 and 10 in all cases, plus a number of posterior association cortices varying across subjects (23). In the present study, we used the same task as in the prior one applied to a group of unmedicated schizophrenic patients to compare their slow potentials with those from the healthy individuals. We focused on the scalp topography of the slow potentials as a first approach to those putative noninvasive indexes of association cortex activity.

We performed two types of statistical comparisons of the topographic data between controls and patients. As a first approach, we used an analysis of a global nature, extracting a topographical index from each individual's whole set of electrodes. Then, we compared each electrode separately to determine which subset of the montage accounted for a possible difference in topography between groups.

\section{Material and Methods}

\section{Subjects}

The sample comprised 20 schizophrenic patients (DSM-IV; 14 males and 6 females, mean age $32.0 \pm 7.9$ years) and 22 healthy controls (14 males and 8 females, mean age $32.4 \pm 9.3$ years). All subjects had normal or corrected to normal vision and hearing. Schizophrenic patients had been drug free for at least 3 weeks, and 4 patients were drug naive. Structured interviews were used, with 
10 patients being classified as paranoid, 6 as residual and 4 as disorganized. Eleven patients were hospitalized, 3 of whom with previous hospitalizations. Psychopathology was assessed by the anchored version of the Brief Psychiatric Rating Scale (BPRS-A) (24) and the Negative Symptoms Rating Scale (NSRS) (25). The mean psychopathological scores were $27.1 \pm 6.6$ in BPRS and $14.8 \pm$ 8.8 in NSRS. Patients were classified into (overlapping) subgroups based both on a categorical distinction (subtype diagnosis from structured interview) and on their ranking on the independent psychopathological dimensions (scores on scales). Mean duration of illness was $11.3 \pm 9.2$ years, and the mean number of previous hospitalizations was $1.2 \pm 1.9$. All subjects were informed about the experiment and signed consent forms approved by the Ethics Committee of the University Hospital.

\section{Stimuli and task}

All aspects of the task were controlled with a commercial computer program (Stim; Neurosoft Inc., El Paso, TX, USA). Visual stimuli subtending less than three degrees in the visual field were presented on a computer screen and formed pairs associated in time, separated by $2.5 \mathrm{~s}$. The three types of pairs comprised randomly intercalated verbal, pictorial and spatial subtasks (words: concrete nouns; abstract visual patterns and traces; central arrows or circles indicating 8 possible positions on the screen). Each stimulus lasted $0.5 \mathrm{~s}$ and the intertrial interval was $6 \mathrm{~s}$. Subjects were required to memorize 8 pairs per block (each presented twice, with reversed order the second time), keeping in mind whether or not there was a categorical match for each pair (food and nonfood verbal task; patterns and traces - pictorial task; same or different position - spatial task). During the test blocks, the same sequence just memorized was presented. However, only the S1 would be initially presented and remained on the screen until the subject decided whether or not its recently learned associated stimulus (S2) belonged to the same category as S1. They had to indicate a match by pressing a button with the right index finger or a mismatch by using the middle finger. After a button press, S1 disappeared from the screen, and was followed, $2.5 \mathrm{~s}$ later, by its corresponding S2. Two and a half seconds after S2, feedback stimuli (S3) were presented. In the present study we only analyzed the slow potentials corresponding to the $5 \mathrm{~s}$ of anticipation of $\mathrm{S} 3$ stimuli during the test trials. The analyses of slow potentials corresponding to the memorization trials, including source reconstruction separated by subtask, were presented elsewhere (26, and Basile LFH, Yacubian J, Castro CC and Gattaz WF, unpublished results). Correct trials were followed by visual S3 stimuli composed of interesting pictures of photographic models and artists that lasted on the screen for $1.5 \mathrm{~s}$, and incorrect trials were followed by an unpleasant sound $(500 \mathrm{~Hz}$, $70 \mathrm{~dB}$ ). A new trial (next S1) was started 3.5 $\mathrm{s}$ after S3 offset. Eighteen blocks were presented to each subject, thus resulting in a total of 288 trials. We measured performance by the percentage of correct trials. Our reasons to use such complex task instead of a motor-type $\mathrm{CNV}$ task are explained in the discussion, and include the fact that the present study is part of a larger project in which we intend to analyze other potentials separately and study other psychiatric conditions (e.g., slow potentials from memorization blocks and evoked potentials).

\section{Recordings, computation of average potentials and topographic analysis}

We used a fast $\mathrm{Ag} / \mathrm{AgCl}$ electrode positioning system consisting of an extended 1020 system, a 123-channel montage (QuikCap; Neuromedical Supplies ${ }^{\circledR}$, El Paso, TX, USA), and an impedance-reducing gel which eliminated the need for skin abrasion (Quik- 
Gel, Neuromedical Supplies ${ }^{\circledR}$ ). Impedance usually remained below $3 \mathrm{kOhms}$, and channels that did not reach these levels were eliminated from the analysis. Two bipolar channels of the 123 channels in the montage were used for recording both horizontal (HEOG) and vertical (VEOG) electro-oculograms. Artifact elimination was automatic: epochs containing signals in either HEOG or VEOG channels above +50 or below $-50 \mu \mathrm{V}$ were eliminated. In our montage, the VEOG detected blinks as deflections above $130 \mu \mathrm{V}$ in the positive direction. In a pilot study, we observed that the magnitudes of small saccades in various directions, directed at stimuli of approximately 3 degrees of eccentricity, were associated with deflections above 70 $\mu \mathrm{V}$ in one or both EOG channels. Therefore, given our $50-\mathrm{Hz}$ low-pass filter and epoch size of $5 \mathrm{~s}$, the $50 \mu \mathrm{V}$ criterion in the EOG channels proved to be empirically sufficient for the total elimination of epochs containing blinks and small eye movements. We used a minimum of 40 epochs of the total of 96 from each task to compute the average slow potentials. Linked mastoids served as reference only for data collection (common average reference was used for mapping), and an electrode anterior to $\mathrm{Fz}(\mathrm{AFz})$ was the ground. The impedances of reference electrodes were measured separately to be within $1 \mathrm{kOhm}$ each, but an internal circuit of the amplifiers summed them (the overall effect of our linked mastoid montage was mostly to lower event-related potential (ERP) amplitudes - with respect to a unilateral montage but also to make topographic maps less prone to distortions from a single hyperactive temporal lobe). We used four 32-channel DC amplifiers (Synamps; Neuroscan Inc., El Paso, TX, USA) for data collection and the Scan 4.0 software package (Neurosoft Inc.) for computation of potential averages. The filter settings for acquisition were from DC to $50 \mathrm{~Hz}$, and the digitization rate was 250 Hz. The electroencephalogram was collected in the continuous mode, and epochs for aver- aging spanned the interval from $300 \mathrm{~ms}$ before button press to $400 \mathrm{~ms}$ after $\mathrm{S} 3$ presentation. Baseline was defined as the $300 \mathrm{~ms}$ preceding button press. Artifact elimination was automatic: epochs corresponding to signals in the EOG channel above +50 or below $-50 \mu \mathrm{V}$ were eliminated. The diagonal EOG detected blinks as deflections of above 130 $\mu \mathrm{V}$ in the positive direction. In a pilot study, we found that the magnitudes of small saccades in response to stimuli of approximately 3 degrees of eccentricity were deflections of above $70 \mu \mathrm{V}$ in either direction. Therefore, given our 50-Hz low-pass filter and epoch size of $5 \mathrm{~s}$, in practice, our $50-\mu \mathrm{V}$ criterion (in either direction) in the EOG channel alone proved to be sufficient for the total elimination of epochs containing blinks and eye movements. We used a minimum of 60 epochs out of a total of 288 to compute the average CNVs. Since the slow potentials presently studied preceded and were related to the feedback anticipation and not to trial outcome itself, both correct and incorrect trials were included in the averages. Our design permitted some subjects who had difficulties to avoid blinking or small eye movements to still produce a sufficient number of uncontaminated epochs. However, even using such criteria, more than 5 patients as well as 3 controls had to be eliminated from the originally recruited group due to artifact contamination (some patients were not able to start the experiment due to restlessness or inability to learn the task instructions).

For comparison purposes, that is, to compute grand averages across subjects that allow subtractions between individual and group data, we retained only the channels devoid of artifacts across all recording sessions. Thus, for computation of topographic maps, our used montage was reduced from 123 original electrodes to 87 good electrodes. The bad electrodes were distributed randomly over the montage, except for a row of the four lowermost occipital sites (neck move- 
ment artifacts), so that no important gaps resulted in the electrode distribution. The electrical data were collapsed across the 22 healthy subjects using the common montage for computation of a grand average control map. To allow for an equal contribution by each subject to the final pattern, where the shape of potential distribution was emphasized (instead of field power, highly variable across subjects), we rescaled each subject's data to an (arbitrary) common power value, choosing the value of the power of the nonscaled, absolute control group average data for this purpose $(2.8 \mu \mathrm{V})$. To visualize possible qualitative differences between groups, we computed a grand average for the patient data as well. We also computed regular grand average maps for performance and for clinical subgroups, separating individuals by the median values for task performance (below or above $71 \%$ for controls and $58 \%$ for patients) and for scores in BPRSpositive symptoms $($ median $=11)$, BPRSdisorganization $($ median $=1.5)$ and NSRS $($ median $=14.5)$.

For visual inspection, all topographic maps were computed in a three-dimensional fashion. The discrete voltage distribution was transformed into a continuous distribution by linear interpolation, and the resulting distribution was projected onto each subject's skin surface. The skin surface was automatically segmented from individual magnetic resonance image (MRI) sets by a commercially available program (Curry V4; Neurosoft Inc.). Group averaged maps were projected onto the segmented skin surface of an individual of median head size. MRIs were obtained by a 1.5 Tesla GE machine model Horizon LX (Walkesha, WI, USA). Image sets consisted of $124 \mathrm{~T}-1$ weighed sagittal images of 256 by 256 pixels spaced $1.5 \mathrm{~mm}$ apart. Acquisition parameters were: standard echo sequence, three dimensions, fast spoiled gradient echo, two excitations, repetition time $=6.6 \mathrm{~ms}$, echo time $=1.6 \mathrm{~ms}$, flip angle of 15 degrees, and field of view $=$
$26 \times 26 \mathrm{~cm}$. Total acquisition time was about 8 min.

For quantitative analysis of the topographic patterns, i.e., of how much each individual scalp distribution of potentials differed from the control pattern, we measured the topographic deviation as the Euclidian distance (quadratic vector norm) on an electrode-by-electrode basis between each subject's rescaled data and the control data. That is, we computed the squared root of the sum of squared differences in voltage between each pair of electrodes (group average minus individual) divided by the number of used channels. Thus, the resulting deviation index was measured in $\mu \mathrm{Vs}$, and by definition was identical to the mean global field power (MGFP) of the potentials obtained by subtraction between individual and group averaged data. MGFP was computed as the squared root of the sum of squared potentials, divided by the number of channels. The rationale for using the control group as a 'normal data bank' reference for comparing patient data could lead to a trivial difference, since the control group was not large and may not be representative of a large population. Thus, we also computed the relative deviation index between each individual and a grand averaged data set obtained from all subjects, controls and patients.

We performed statistical analysis (Student $t$-test) to compare the deviation index, MGFP and task performance across the patient and healthy control groups. We also computed Pearson's correlation coefficients between these physiological indexes and the psychopathological measures (NSRS, BPRS and subscales). Finally, we performed a multivariate statistical analysis (MANOVA, with electrodes as dependent variables and group as fixed factor) using the potential values at all electrode locations in the montage, to determine whether particular regions (electrode subsets) accounted for possible group differences. 


\section{Results} Task performance and mean global field
power

Individual reports on task difficulty were variable, in agreement with performance scores. Average performance $( \pm$ standard deviation) was $72.4 \pm 11.8 \%$ for the control group and $59.7 \pm 10.1 \%$, for the patient group. The difference in performance was statistically significant $(\mathrm{P}=0.001)$. Performance was significantly correlated with total BPRS score $(\mathrm{r}=-0.472, \mathrm{P}=0.036)$, with NSRS $(r=-0.524, P=0.018)$ and with BPRS-subscore affective blunting/negative symptoms $(r=-0.454, P=0.044)$. We divided the patients into pairs of subgroups based on high versus low scores on each psychopathological scale, and tested whether mean performance was different between subgroups using the Student $t$-test. Only the subgroups defined by a total BPRS score differed significantly in performance (high BPRS subgroup: mean performance $=54.4 \pm$ $6.00 \%$; low BPRS subgroup: mean performance $=64.2 \pm 11.0 \% ; \mathrm{P}=0.023)$.

All subjects presented slow potentials, regardless of task performance, although three patients had potentials of very low amplitudes (MGFP below $3 \mu \mathrm{V}$ ). The average MGFP was $5.8 \pm 2.3 \mu \mathrm{V}$ for the control group and $5.1 \pm 2.2 \mu \mathrm{V}$ for the patient group, at the slow potential peak amplitude $(5.0 \mathrm{~s}$ after button press, or at the moment of S3 onset), and this difference was not significant. Signal-to-noise ratios, initially computed on a channel-wise basis (from the 300 ms baselines) and then as an average across channels, were also similar and not significantly different between groups (7.88 and 7.35, respectively). Among subgroups of patients, divided by scores on BPRS and NSRS, MGFP was significantly different only between subgroups of high and low scores on BPRS for positive symptoms $(6.0 \pm 1.5$ and $4.11 \pm 0.9 \mu \mathrm{V}$, respectively; $\mathrm{P}=0.05)$.

\section{Slow potential topography at visual inspection}

The main finding and focus of the present study concerned the spatial distribution of slow potentials. Whereas most healthy subjects presented a simple pattern of potential extrema, with maximum positive voltage close to the $\mathrm{C} 3$ electrode and maximum negative close to FP2, the patients presented a more complex pattern, with a higher degree of variability in slow potential topography. Typically, in patients, the regions of extrema, especially the positive one, were fragmented into many disconnected minor peaks, with a more folded, sinusoidal appearance of the zero isopotential contour lines. Such fragmentation of potential extrema can be visualized on maps computed from either individual or group averaged data (Figure 1). Due to the more complex appearance of patient maps, we counted the total number of disconnected positive voltage regions in each subject. There was no significant difference in such numbers between groups. Therefore, we concluded that the difference in maps between groups was due to the shape and not to the number of same polarity regions, with patients having more interdigitation between positive and negative regions.

We also analyzed the appearance of grand averages resulting from the division of patient and control groups into subgroups based on performance and on scores on BPRS (subscores for positive symptoms and disorganization) and NSRS. In controls, the maps obtained from high and low task performance subgroups were similar in topography. In patients, the maps from both low and high task performance subgroups showed the fragmented pattern (Figure 2). In all cases, maps from patient subgroups deviated from the control map, sharing the fragmented pattern. Among all patient subgroups, the maps corresponding to high BPRS subscores were more grossly fragmented and presented high positive voltage in the right hemisphere. 

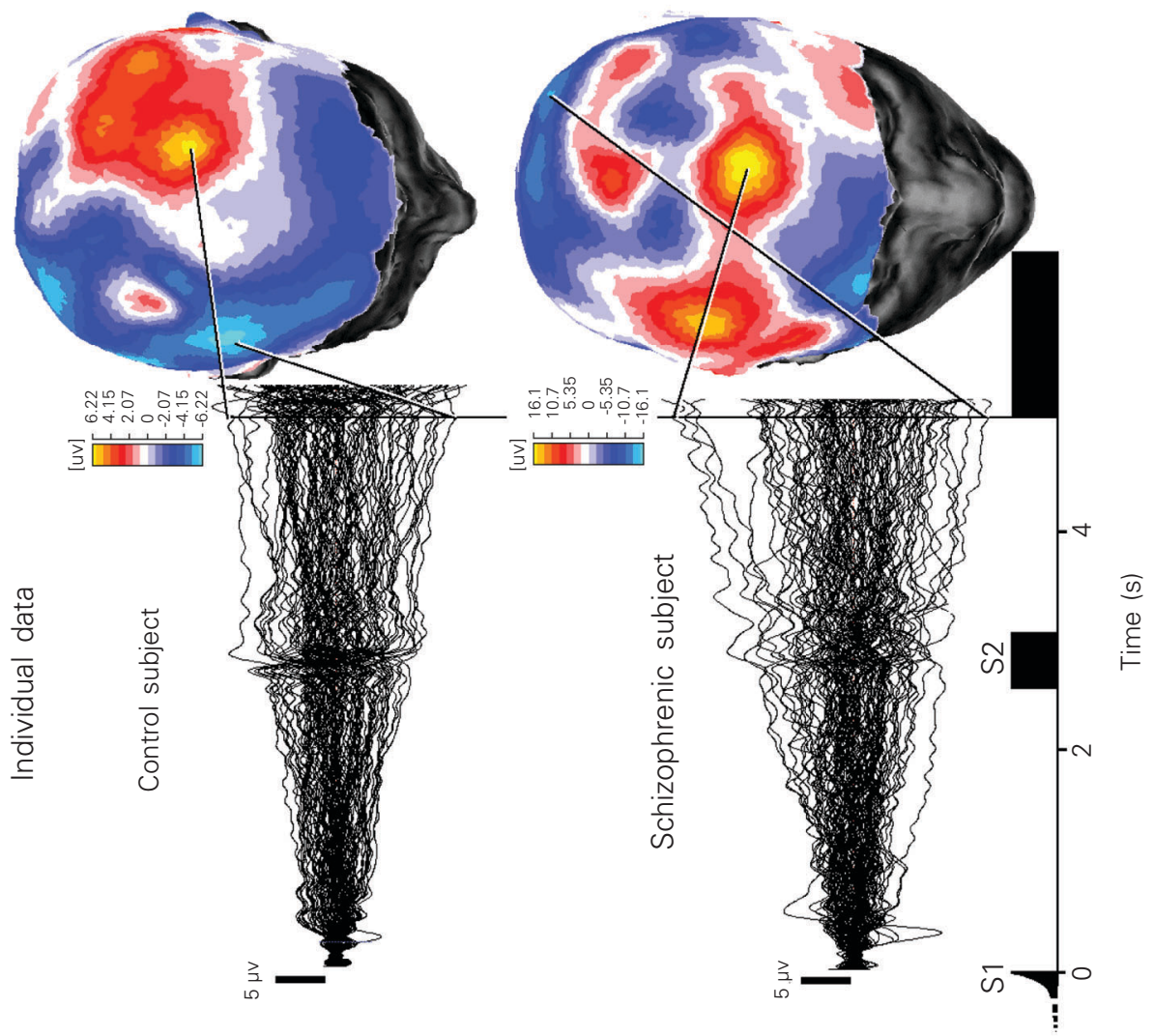

Figure 1. Group averages and individual examples of slow potentials and corresponding isopotential contour maps. Maps shown were at the onset of S3 performance feedback stimulus. Angle of view: $40^{\circ}$ from the nasion-right-left preauricular plane. Maps were projected on the skin surface and were automatically segmented from individual magnetic resonance images. Group average maps were normalized so that each individual contributed equally with respect to electrical power, and were projected onto the segmented skin of a subject with medium head size. Numbers in color scale: voltage in $\mu \mathrm{V}$.
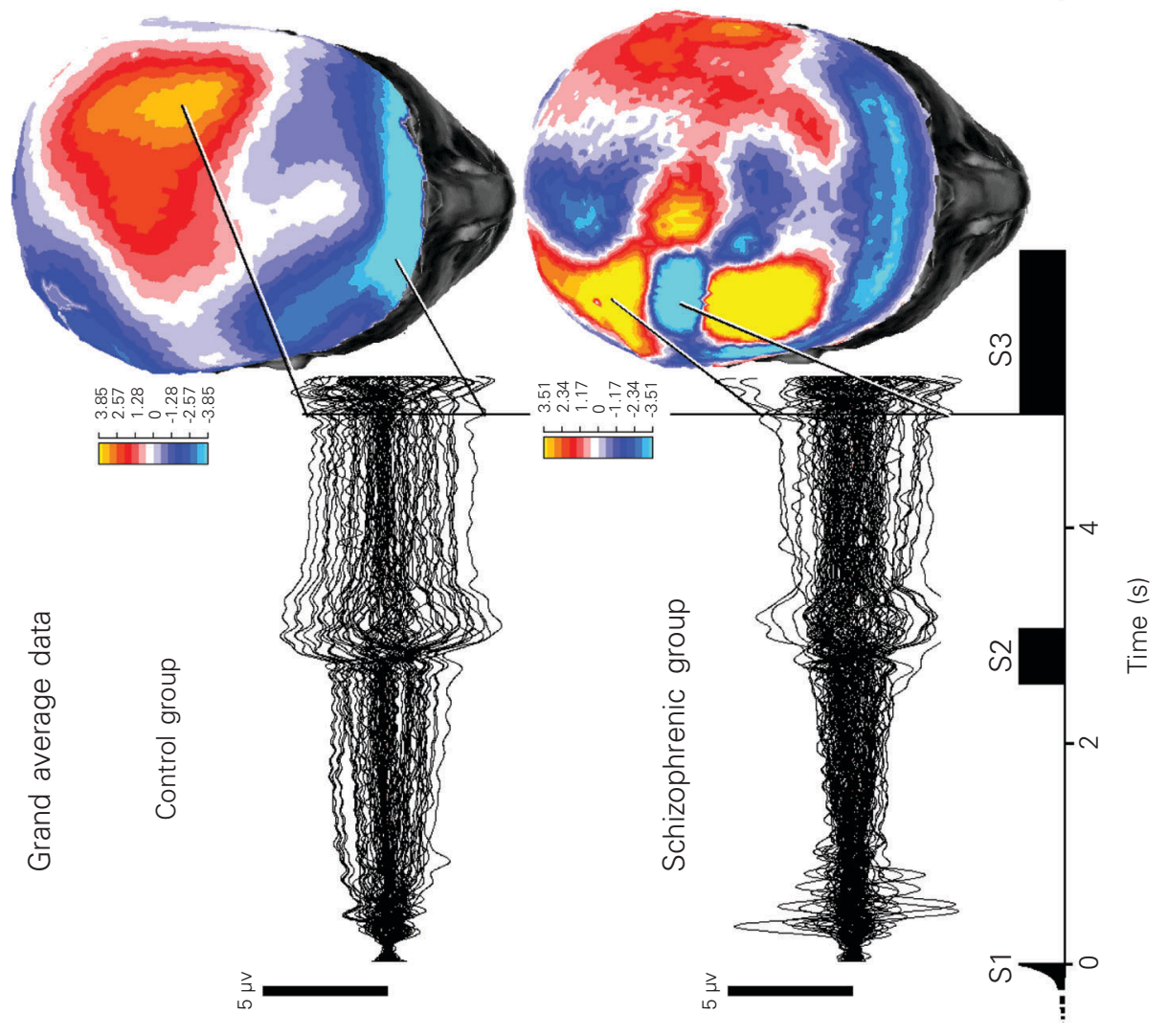
Figure 2. Topographic maps from group averaged data corresponding to subgroups of patients with task-performance above (left, labeled good) and below the median (right, labeled poor). Scales are as in Figure 1. At first impression, the good performance average map resembles the control group map from Figure 1, but notice the low potential gradients on the right hemisphere between the voltage extrema.

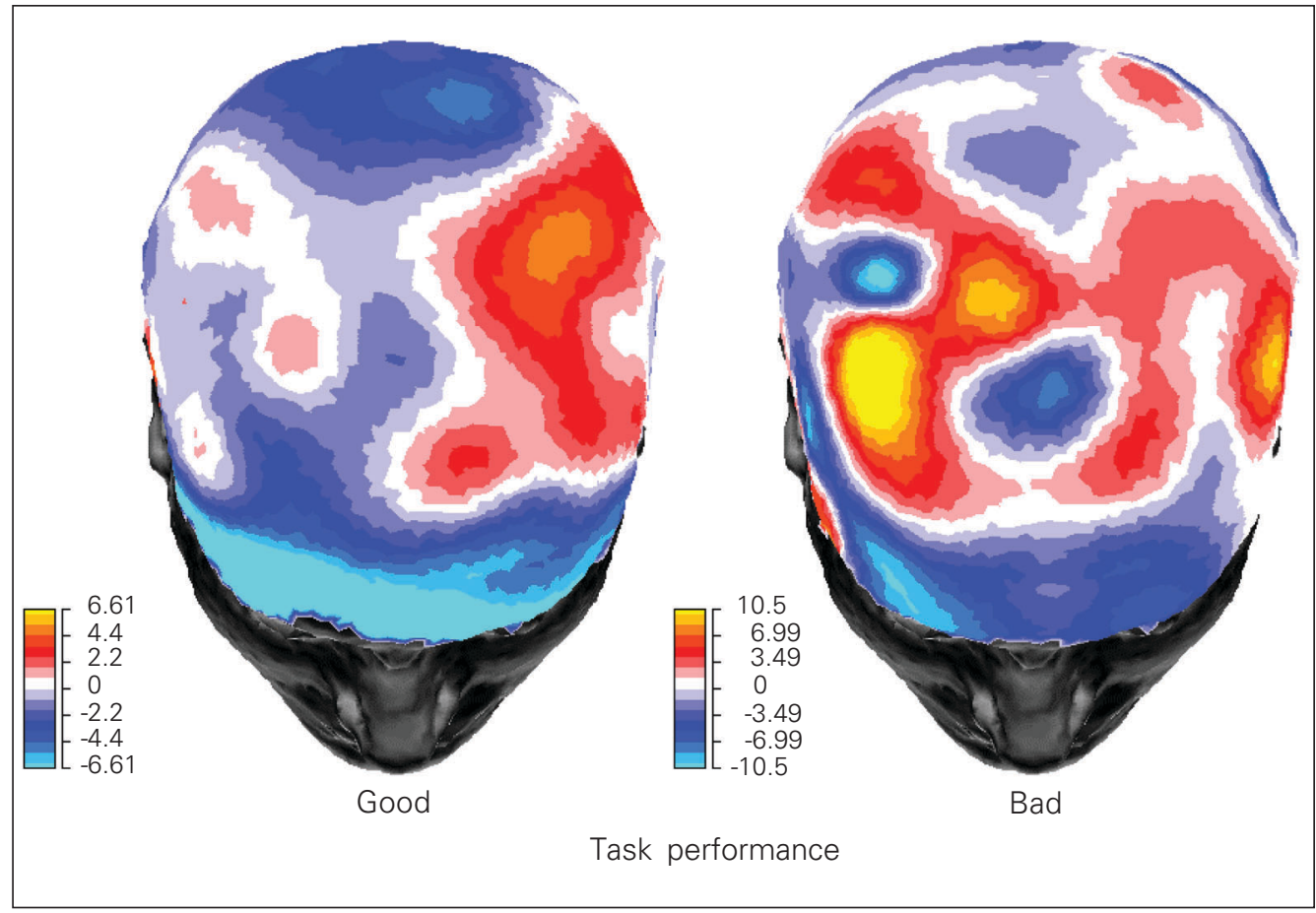

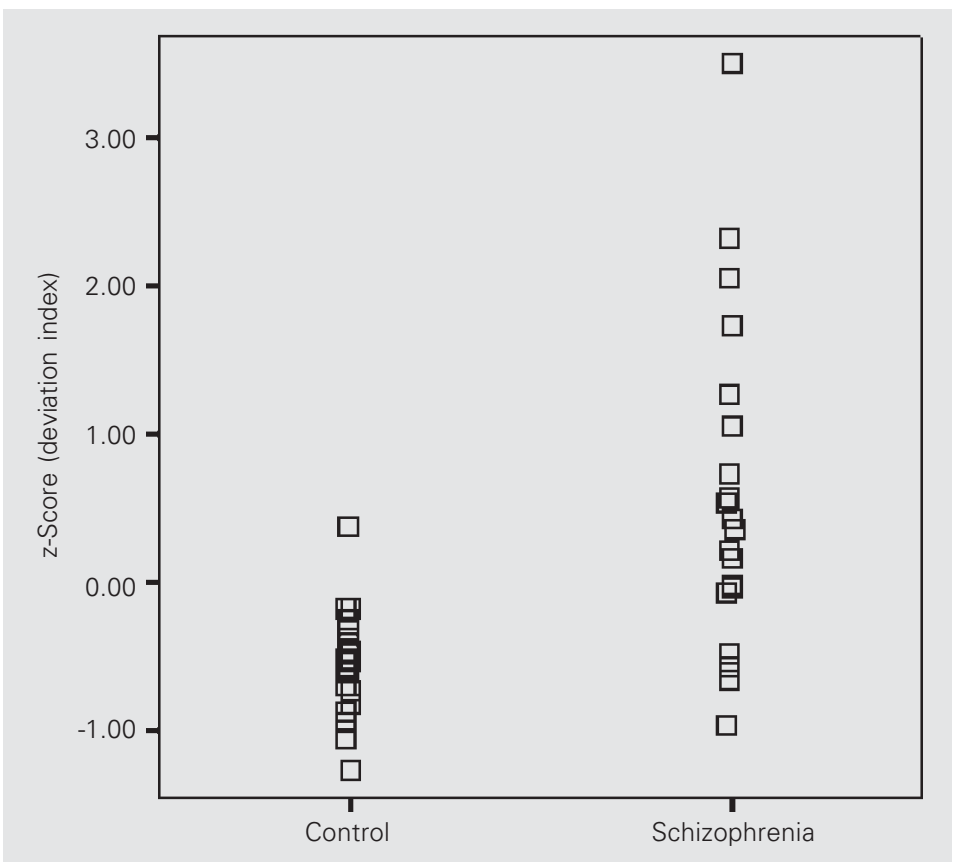

Figure 3. Topographic deviation scatterplot, with electrical power (originally in $\mu \mathrm{V}$ ) transformed into z-scores. Each score was obtained from the root mean square of the channel-by-channel differences between individual slow potential amplitude and the group averaged potentials (controls plus patients). The control group consisted of 22 subjects and the schizophrenic group of 20 patients.

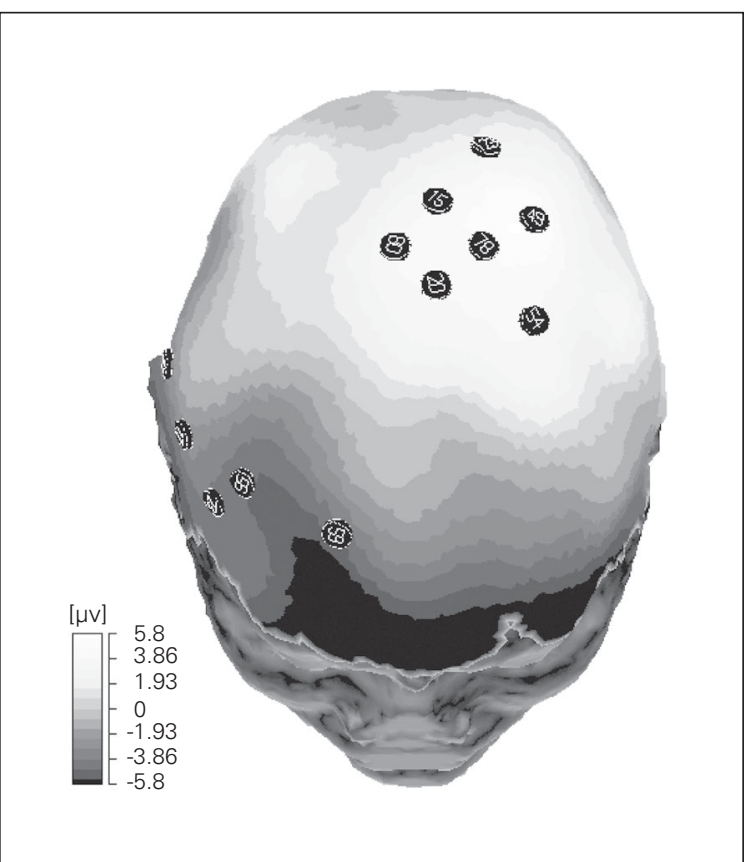

Figure 4. Electrode positions where statistically significant differences in mean voltage between patients and controls were observed $(P<0.01$, MANOVA). The fronto-central set was centered at the left of FCz and the frontotemporal set at FT8. Electrodes were plotted on the original control group average map to show relation with regions of high slow potential voltage. 


\section{Topographic deviation index}

The deviation of individual electrical data from the control group averaged data was tabulated in the form of the topographic deviation index, as described in Methods, for group and subgroup comparisons. The mean topographic deviation was higher in patients than in controls $(\mathrm{P}<0.001)$. When topographic deviation was computed with respect to grand averaged data obtained from the complete set of subjects (controls plus patients), the resulting individual indexes were virtually indistinguishable from those obtained using only the control subjects as a reference. Typically, differences in the indexes (electrical power) were not altered beyond $0.2 \mu \mathrm{V}$ for any given individual, with respect to the method using only the controls as the 'normal data bank'. In this case also, average indexes were significantly different between controls and patients $(\mathrm{P}<$ 0.001). The resulting deviation indexes are presented in Figure 3, with electrical power in $\mu \mathrm{V}$ transformed into z-scores. We also computed correlations between MGFP and deviation index with scores on the BPRS (including subscores) and NSRS. There was a significant correlation between topographic deviation and performance $(r=-0.358, P=0.020)$, but not with scores on the psychopathological scales (the correlation between topographic deviation and the BPRS subscore for thought disorder almost reached significance: $\mathrm{r}=0.430, \mathrm{P}=$ 0.058). Finally, we divided the group of patients into halves, i.e., subgroups defined by high versus low task performance and high versus low scores on psychopathology, to test whether any pair of subgroups significantly differed in topographic deviation. We found that topographic deviation was significantly different between subgroups of high and low scores on BPRS for positive symptoms ( $\mathrm{P}=$ 0.042; Table 1).

\section{Individual electrode analysis}

We performed a MANOVA to test for differences in mean slow potential amplitudes in individual electrodes across the two groups. By using a significance cutoff level of $\mathrm{P}=0.01$, a simple spatial pattern of electrodes emerged as the subset differing across groups. They were grouped in only two places, 7 in the (left) fronto-central and 4 in the right frontotemporal regions. The first region was centered at the left of the electrode between $\mathrm{Fz}$ and $\mathrm{Cz}(\mathrm{FCz})$ and the second at a right frontotemporal electrode (FT8). In all cases, the electrodes were those corresponding to high voltage positions from the control map, following a line of highest overall voltage gradient (Figure 4). In all cases, the differences in voltage were in the form of low amplitudes on the patients' average potentials as compared to the control data. That is, patients had low electrical power on the (left) fronto-central and right frontotemporal regions.

\section{Discussion}

We observed slow potentials in the recordings from all individuals. During trials, when a given subject did not remember a stimulus paired-association, the task would be reduced to mere guessing and anticipation of the feedback stimuli: subjects had at least to initiate trials at will, and wait without

Table 1. Topographic deviation index, in $\mu \mathrm{V}$, for subgroups of patients defined by task performance and psychopathology scores.

\begin{tabular}{lcc}
\hline Subgroup & High & Low \\
\hline Performance & $3.8 \pm 1.0$ & $4.5 \pm 1.5$ \\
NSRS & $3.9 \pm 0.9$ & $4.3 \pm 1.6$ \\
BPRS total & $4.7 \pm 1.5$ & $3.6 \pm 0.6$ \\
BPRS-positive symptoms & $4.5 \pm 1.5$ & $3.8 \pm 0.9$ \\
BPRS disorganization & $4.6 \pm 1.4$ & $3.7 \pm 1.0$
\end{tabular}

Patients were divided, in each case using the median performance or score value, into two halves containing subjects scoring above (high) or below (low) the median values. Data are reported as means \pm SD. BPRS and NSRS $=$ Brief Psychiatric and Negative Symptoms Rating Scales, respectively. 
blinking for each outcome. Thus, it is important to insist that the present potentials were not related to task performance (correct versus incorrect outcomes), and especially not related to remembering each pair of stimuli since remembering their categorical relation was sufficient for correct performance. The slow potentials corresponded to the anticipation of the feedback stimuli, and not to the presented feedback, since they preceded each S3. However, we chose the present task for additional reasons: by requiring button presses at the start of the trials, we avoided the presence of movement-related fields on our recordings, so that the slow potentials would predominantly reflect association cortex activity $(17,23)$; second, some schizophrenic patients may functionally resemble patients with medial frontal lesions, who are known to have low performance on 'experimental gambling', which has a guessing aspect similar to our task $(27,28)$; finally, we originally expected that both the guessing and the anticipation of the particular type of S3 stimuli that we chose could engage these medial, phylogenetically older prefrontal areas. That is, the S3 stimuli, both for signaling trial outcomes and for being interesting in themselves, were expected to recruit prefrontal areas more densely interconnected with structures such as the expanded amygdala. However, according to our recent current density reconstruction results, only areas on the prefrontal convexity were active, a result that we interpret to be due to the insufficiently arousing or biologically 'relevant' nature of the stimuli (23).

The present type of slow potential provoking task thus appears to be well suited for patients, since task performance is not strictly related to either the presence or topography of the potentials. We did expect more difficulties for the patients due to primary memory deficits, which are believed to be part of a general intellectual impairment in schizophrenia (29-31). In our sample, some patients fluctuated in time with respect to keep- ing in mind the task goal, others were restless or clearly delusional, and two had important thought disorder. In spite of these difficulties, which were more important than remembering the pairs of stimuli, signal to noise ratios and mean global field power were comparable across groups. Only three patients had potentials of very low amplitude.

The most important finding of the present study was the difference between schizophrenics and controls regarding the spatial pattern of slow potentials over the scalp. Regions of voltage extrema over the scalp in schizophrenics were fragmented and of increased complexity when compared to the control pattern. This could be observed in most cases by visual inspection of isopotential maps, and was reflected in the topographic deviation index, whose mean value was significantly higher for the patient group. Moreover, the position of the highest electrical gradients (usually close to the zero isopotential lines) was more anterior in patients than in controls. This may be equivalent to the findings of a more anterior conventional CNV maximum in schizophrenic patients in studies using few electrodes and reference to particular electrodes $(32,33)$. It is possible that some controversies in the literature regarding the topography of event-related potentials, for instance P3s or conventional CNVs, may be clarified by the use of highdensity sensory arrays, as used in the present study. If in the present study fewer electrodes had been used, it is likely that a wide variability in potential amplitude would have been observed, as usually reported in the literature. This was due to spatial undersampling of the complex potential distribution of patients; for instance, different choices for analysis among neighbor electrodes from the 10-20 system can lead to opposite results for a given patient.

It is of interest to emphasize that the topographic deviation observed in our patients was independent of psychopathologi- 
cal status, clinical subtypes or task performance. By computing grand averaged maps for subgroups of patients, divided by the median values of task performance or of psychopathological scores, we found that all subgroups still presented the fragmentation of voltage extrema and altered position of highest gradients. This independence was also reflected in the lack of significant correlations between the topographic deviation index and either NSRS, BPRS (including its subscores) or task performance. However, we found a significant difference in topographic deviation between subgroups of high versus low scores on BPRS for positive symptoms. This may indicate that, although topographic fragmentation does not correlate with any psychopathological dimension of analysis, patients with predominantly positive symptoms are the ones who exhibit it not exclusively but more strongly. Because our patients were unmedicated or drug naive, it is unlikely that the topographic fragmentation of slow potentials was secondary to a nonspecific drug artefact. Further longitudinal studies are necessary to determine whether the fragmentation observed may be seen as a trait marker of schizophrenia.

Only two regions on the scalp contained the electrodes that had significantly different voltages at the slow potential peak across patients and controls, namely, the (slightly left) fronto-central area containing the slow potential-positive voltage peak and the right frontotemporal area corresponding to part of the high negative area. The overall lower slow potential electrical power in such frontal scalp regions in schizophrenia is compatible with the 'hypofrontality' idea. However, one should not expect a trivial correspondence between the (group) overall scalp regions of low voltages and individual patterns of intracranial generators.

Various underlying facts could explain the topographic findings in schizophrenia. We speculate that the large scale functional cortical areas, whose sustained electrical activity is reflected as scalp slow potentials, present a lower definition in patients, due to a lower cohesion with each other or with subcortical cell fields and nuclei (such as the cholinergic or dopaminergic ascending projections). The latter may be the very structures that define cortical functional areas, i.e., dynamic ensembles of areas established after task learning. Alternatively, the generators of average slow potentials in patients may be simply more dispersed, possibly due to a loss of normal cortico-cortical connectivity, as predicted from models of abnormal cortical pruning (34). Finally, it is also possible that an overall different set of areas become active when patients perform a given task, as compared to healthy subjects. These hypotheses might be confirmed or refuted after the development of quantitative methods to compare our source reconstruction results across groups. The available quantitative methods (computation of current density vector sums restricted to chosen percentile bands of current distribution) when applied to the reconstruction results of the present data from patients (results not shown), did not distinguish the groups in a statistically significant way. However, at visual inspection, they are in agreement with the dispersion hypothesis. We are currently analyzing the generators of long-latency endogenous evoked potentials corresponding to the three subtasks, and have already observed a significantly lower focality (current density/volume occupied by currents) in patients.

Finally, we must stress that, although the generators of the slow potentials of the present type are complex in distribution even in healthy individuals $(23,26)$, and did not allow for a quantitative demonstration of the dispersion hypothesis, the present study must be interpreted as indirect evidence in favor of an abnormality in multiple cortical association areas in schizophrenia. This agrees with a growing body of independent evidence against a mere 'hypofrontality' hypo- 
thesis. Neuropathological, anatomical and functional studies have emphasized such widespread nature of cortical alterations (3538). In particular, the concept of decreased prefrontal activity in schizophrenia itself is being revised: metabolic tracing studies (fMRI, PET and SPECT) show opposite results depending especially on task conditions (for a review, see Ref. 35). Our own recent reconstruction results show both hyper- and hypoactive cortical generators of slow potentials in patients (Basile LFH, Yacubian J, Castro CC and Gattaz WF, unpublished results). Thus, electrical and metabolic dysfunction in multiple cortical areas (with neuropsychological loss) is what one would expect from the progressive tissue loss observed in anatomical longitudinal studies using high-resolution MRI (36). The dis- tributed cortical abnormalities in schizophrenia, in turn, may reflect a still larger scale set of encephalic alterations, including the thalamo-cortical-basal ganglium circuits and their interconnections with the dopaminergic projections, according to findings from neuropathological studies $(37,38)$.

\section{Acknowledgments}

We wish to thank Dr. Cláudio C. de Castro (Incor, FM, University of São Paulo) for providing the NMR images for all subjects, and Dr. Milkes Y. Alvarenga and Prof. Marcus V. Baldo (Department of Physiology and Biophysics, ICB, University of São Paulo) for their critical suggestions about data analysis.

\section{References}

1. Gattaz WF, Mayer S, Ziegler P, Platz M \& Gasser T (1991). Hypofrontality on topographic EEG in schizophrenia. European Archives of Psychiatry and Clinical Neuroscience, 241: 328-332.

2. Sponheim SR, Clementz BA, lacono WG \& Beiser M (1994). Resting EEG in first-episode and chronic schizophrenia. Psychophysiology, 31: $37-43$

3. Fehr $T$, Kissler J, Moratti $S$, Wienbruch $C$, Rockstroh B \& Elbert $T$ (2001). Source distribution of neuromagnetic slow waves and MEGdelta activity in schizophrenic patients. Biological Psychiatry, 50: 108-116.

4. Clementz BA \& Blumenfeld LD (2001). Multichannel electroencephalographic assessment of auditory evoked response suppression in schizophrenia. Experimental Brain Research, 139: 377-390.

5. Verleger R (1988). Event-related potentials and cognition: A critique of the context updating hypothesis and an alternative interpretation of P3. Behavioral and Brain Sciences, 11: 343-427.

6. Basile LFH, Rogers RL, Simos PG \& Papanicolaou AC (1997). Magnetoencephalographic evidence for common sources of long latency fields to rare target and rare novel stimuli. International Journal of Psychophysiology, 25: 123-137.

7. Blackwood DH, Whalley LJ, Christie JE, Blackburn IM, St Clair DM \& Mclnnes A (1987). Changes in auditory P3 event-related potential in schizophrenia and depression. British Journal of Psychiatry, 150: 154-160.

8. Louzã MR \& Maurer K (1989). Differences between paranoid and nonparanoid schizophrenic patients on the somatosensory P300 event-related potential. Neuropsychobiology, 21: 59-66.

9. Pfefferbaum A, Ford JM, White PM \& Roth WT (1989). P3 in schizophrenia is affected by stimulus modality, response requirements, medication status, and negative symptoms. Archives of General Psychiatry, 46: 1035-1044.

10. Niwa S, Hiramatsu K, Saitoh O, Fukuda M, Kameyama T, Itoh K \&
Hayashida S (1992). Information dysregulation and event-related potentials in schizophrenia. Schizophrenia Bulletin, 18: 95-105.

11. Iwanami A, Kanamori R, Isono H, Okajima Y \& Kamijima K (1996). Impairment of inhibition of unattended stimuli in schizophrenic patients: event-related potential correlates during selective attention. Neuropsychobiology, 34: 57-62.

12. McCallum WC (1988). Potentials related to expectancy, preparation and motor activity. In: Picton TW (Editor), Handbook of Electroencephalography and Clinical Neurophysiology. Human Event-Related Potentials. Revised series, Vol. 3. Elsevier Science Publishers, Kidlington, Oxford, UK, 427-534.

13. Walter WG, Cooper R, Aldridge VJ, McCallum WC \& Winter AL (1964). Contingent negative variation: an electric sign of sensorimotor association and expectancy in the human brain. Nature, 203: 380-384.

14. Basile LFH, Rogers RL, Bourbon WT \& Papanicolaou AC (1994). Slow magnetic fields from human frontal cortex. Electroencephalography and Clinical Neurophysiology, 90: 157-165.

15. Basile LFH, Simos PG, Brunder DG, Tarkka IM \& Papanicolaou AC (1996). Task-specific magnetic fields from the left human frontal cortex. Brain Topography, 9: 31-37.

16. Basile LFH, Brunder DG, Tarkka IM \& Papanicolaou AC (1997). Magnetic fields from human prefrontal cortex differ during two recognition tasks. International Journal of Psychophysiology, 27: 29-41.

17. Bocker KB, Brunia $\mathrm{CH}$ \& van den Berg-Lenssen MM (1994). A spatiotemporal dipole model of the stimulus preceding negativity (SPN) prior to feedback stimuli. Brain Topography, 7: 71-88.

18. Tarkka IM \& Basile LFH (1998). Electrical source localization adds evidence for task-specific CNVs. Behavioural Neurology, 11: 21-28.

19. Rosahl SK \& Knight RT (1995). Role of the prefrontal cortex in generation of the contingent negative variation. Cerebral Cortex, 2: 
123-134

20. Zappoli R, Zappoli F, Versari A, Arnetoli G, Paganini M, Arneodo MG, Poggiolini D \& Thyrion EZ (1995). Cognitive potentials: ipsilateral corticocortical interconnections in prefrontal human cortex ablations. Neuroscience Letters, 193: 140-144.

21. Ikeda A, Luders HO, Collura TF, Burgess RC, Morris HH, Hamano T \& Shibasaki H (1996). Subdural potentials at orbitofrontal and mesial prefrontal areas accompanying anticipation and decision making in humans: a comparison with Bereischaftspotential. Electroencephalography and Clinical Neurophysiology, 98: 206-212.

22. Hamano T, Luders HO, Ikeda A, Collura TF, Comair YG \& Shibasaki $H$ (1997). The cortical generators of the contingent negative variation in humans: a study with subdural electrodes. Electroencephalography and Clinical Neurophysiology, 104: 257-268.

23. Basile LFH, Ballester G, Castro CC \& Gattaz WF (2002). Prefrontal cortex activity assessed by high-resolution EEG and current density reconstruction. International Journal of Psychophysiology, 45: 227240.

24. Woerner MF, Manuzza S \& Kane JM (1988). Anchoring the BPRS: An aid to improved reliability. Psychopharmacology Bulletin, 24: 112-117.

25. lager AC, Kirsh DG \& Wyatt RJ (1985). A negative symptom rating scale. Psychiatry Research, 16: 27-36.

26. Basile LFH, Baldo MV, Castro CC \& Gattaz WF (2003). The generators of slow potentials obtained during verbal, pictorial and spatial tasks. International Journal of Psychophysiology, 48: 55-65.

27. Eslinger PJ \& Damasio AR (1985). Severe disturbance of higher cognition after bilateral frontal lobe ablation: Patient EVR. Neurology, 35: 1731-1741.

28. Damasio AR (1996). The somatic marker hypothesis and the possible functions of the prefrontal cortex. Philosophical Transactions of the Royal Society of London, Series B. Biological Sciences, 351: 1413-1420.
29. Stip E (1996). Memory impairment in schizophrenia: perspectives from psychopathology and pharmacotherapy. Canadian Jounal of Psychiatry, 41 (Suppl 2): S27-S34.

30. Heinrichs RW \& Zakzanis KK (1998). Neurocognitive deficit in schizophrenia: a quantitative review of the evidence. Neuropsychology, 12: 426-445.

31. Hawkins KA (1999). Memory deficits in patients with schizophrenia: preliminary data from the Wechsler Memory Scale - Third Edition support earlier findings. Journal of Psychiatry and Neuroscience, 24: 341-347.

32. Rockstroh B, Müller M, Wagner M, Cohen R \& Elbert T (1994). Event-related and motor responses to probes in a forewarned reaction time task in schizophrenic patients. Schizophrenia Research, 13: 23-34.

33. Wagner M, Rendtorff N, Kathmann N \& Engel RR (1996). CNV, PINV and probe-evoked potentials in schizophrenics. Electroencephalography and Clinical Neurophysiology, 98: 130-143.

34. Hoffman RE \& Dobscha SK (1989). Cortical pruning and the development of schizophrenia: a computer model. Schizophrenia Bulletin, 15: 477-490.

35. Manoach D (2003). Prefrontal cortex dysfunction during working memory performance in schizophrenia: reconciling discrepant findings. Schizophrenia Research, 60: 285-298.

36. Thompson PM, Vidal C, Giedd JN, Gochman P, Blumenthal J, Nicolson R, Toga AW \& Rapoport JL (2001). Mapping adolescent brain change reveals dynamic wave of accelerated gray matter loss in very early-onset schizophrenia. Proceedings of the National Academy of Sciences, USA, 98: 11650-11655.

37. Arnold SE \& Trojanowski JQ (1996). Recent advances in defining the neuropathology of schizophrenia. Acta Neuropathologica, 92: 217-231.

38. Powers RE (1999). The neuropathology of schizophrenia. Journal of Neuropathology and Experimental Neurology, 58: 679-390. 